\title{
Bi: Perfect surfactant for Ge growth on Si(111)?
}

\author{
T. Schmidt, ${ }^{\text {a) }}$ J. Falta, ${ }^{\text {b) }}$ and G. Materlik \\ Hamburger Synchrotronstrahlungslabor HASYLAB am Deutschen Elektronen-Synchrotron DESY, \\ Notkestrasse 85, D-22607 Hamburg, Germany \\ J. Zeysing, G. Falkenberg, and R. L. Johnson \\ II. Institut für Experimentalphysik, Universität Hamburg, D-22761 Hamburg, Germany
}

(Received 16 November 1998; accepted for publication 12 January 1999)

\begin{abstract}
We have investigated the growth of $\mathrm{Ge}$ on Bi-terminated $\mathrm{Bi}: \mathrm{Si}(111)-\sqrt{3} \times \sqrt{3}$. In-situ measurements of x-ray standing waves, crystal truncation rods and scanning tunneling microscopy clearly show that, at substrate temperatures around $485^{\circ} \mathrm{C}$, smooth and homogeneous $\mathrm{Ge}$ films of thicknesses exceeding 30 bilayers $\mathrm{Ge}$ can be grown. For Ge coverages larger than 10 bilayers, the Ge film is completely relaxed. $\mathrm{Bi}$ is found to segregate to the surface during Ge deposition, and can be removed from the surface after growth by mild annealing at $520^{\circ} \mathrm{C}$ as proven by Auger electron spectroscopy. (C) 1999 American Institute of Physics. [S0003-6951(99)03310-0]
\end{abstract}

Surfactant mediated epitaxy (SME) ${ }^{1}$ of semiconductor structures has attracted considerable interest recently because it enables the growth of structures which are not achievable by conventional molecular beam epitaxy (MBE) or chemical vapor deposition (CVD). For growth of $\mathrm{Ge}$ on $\mathrm{Si}(111)$ so far $\mathrm{Sb}^{2}$ and $\mathrm{Ga}^{3-5}$ have been reported to successfully act as surfactants. The use of $\mathrm{Ga}$ is accompanied by the formation of stacking faults ${ }^{3}$ and is of little technological interest. However, growth of Ge on the low Ga coverage $\sqrt{3} \times \sqrt{3}$ surface may be interesting for intentional lateral patterning. ${ }^{4}$ Surfactant mediated epitaxy of $\mathrm{Ge}$ on $\mathrm{Si}(111)$ is especially promising for the production of high speed devices due to the existence of an effective relaxation mechanism producing a dislocation network at the Si-Ge interface ${ }^{6}$ and a defect free Ge film for optimum device quality. However, so far most work has concentrated on $\mathrm{Sb}$ as a surfactant. ${ }^{2,6-8}$

Here we present the results of a detailed study on the properties of $\mathrm{Ge}$ growth on $\mathrm{Bi}$-terminated $\mathrm{Si}(111)$ substrates. Although Bi has been shown to fail as a surfactant for growth temperatures as low as $300^{\circ} \mathrm{C},{ }^{9}$ we will show that at higher temperatures around $485^{\circ} \mathrm{C}$ Bi enables growth of very smooth and homogenous Ge films. A major drawback of SME is the incorporation of the surfactant in the growing film, which results in an undesirable doping of the Ge layer. Because of the very low solid solubility of $\mathrm{Bi}$ in $\mathrm{Si}$ and $\mathrm{Ge}$ (about three orders of magnitude lower than for $\mathrm{Sb}$ ), very low dopant concentrations are expected for Bi-SME. Furthermore, we will show that $\mathrm{Bi}$ can be removed effectively from SME grown films by mild annealing at $520^{\circ} \mathrm{C}$.

The samples were prepared in an ultrahigh vacuum (UHV) system with a base pressure of $3 \times 10^{-11} \mathrm{mbar}$, equipped with a $\mathrm{Ge}$ and a Bi Knudsen cell as well as a low energy electron diffraction (LEED) system and a cylindrical mirror analyzer for Auger electron spectroscopy (AES). After preparation, the samples were transferred under UHV conditions into portable UHV chambers. These chambers had a base pressure of $5 \times 10^{-10} \mathrm{mbar}$ or better and were

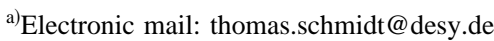

${ }^{b)}$ Electronic mail: falta@desy.de
}

equipped with $400 \mu \mathrm{m}$ thick Be windows, allowing x-ray experiments. Scanning tunneling microscopy (STM) measurements were performed in a separate UHV system. For $\operatorname{Si}(111)$ substrates $10 \times 10 \times 2 \mathrm{~mm}^{3}$ crystals were used. After polishing and RCA chemical cleaning, the samples were introduced to UHV and degassed for at least $24 \mathrm{~h}$ at $600^{\circ} \mathrm{C}$ in order to remove any contamination. For x-ray standing wave (XSW) experiments, special care was taken to avoid any damage to the bulk crystal structure. Therefore the temperature was varied at a rate $<1{ }^{\circ} \mathrm{C} / \mathrm{s}$ only. $\mathrm{Si}(111)-7 \times 7$ surfaces were reproducibly prepared by annealing at $850-900{ }^{\circ} \mathrm{C}$ for $5-10 \mathrm{~min}$. Prior to Ge deposition, Bi was adsorbed onto the surface at $485^{\circ} \mathrm{C}$. This resulted in the formation of the $\mathrm{Bi}: \operatorname{Si}(111)-\sqrt{3} \times \sqrt{3}-\beta$ surface. This structure consists of $\mathrm{Bi}$ in $T_{1}$ sites at a saturation coverage of $2 / 3 \mathrm{ML}^{10}\left(1\right.$ monolayer $\left.(\mathrm{ML})=7.83 \times 10^{14} \mathrm{~cm}^{-2}\right)$. During Ge deposition the Bi flux of 1-2 ML/min was maintained in order to compensate for $\mathrm{Bi}$ desorption which is not negligible at this growth temperature. As on $\mathrm{Si}, \mathrm{Bi}$ occupies $T_{1}$ sites on top of Ge, as shown by XSW at low ( $\lesssim 1 \mathrm{ML}) \mathrm{Ge}$ coverages. ${ }^{11}$

The lattice constant of both a pseudomorphically strained as well as a relaxed Ge film is larger than the lattice constant of Si. Therefore in XSW one expects a decreasing contrast in the fluorescence yield with increasing film thickness. ${ }^{12}$ For the (111) Fourier component of the Ge distribution function $A_{111}$ the calculation gives

$$
\begin{aligned}
A_{111} & =\frac{1}{N} \eta \sum_{n=0}^{N-1} \exp \left(2 \pi i\left(n \delta+\delta_{0}\right)\right) \\
& =\frac{1}{N} \eta \frac{\sin \left(2 \pi N \frac{\delta}{2}\right)}{\sin \left(2 \pi \frac{\delta}{2}\right)} \exp \left(2 \pi i\left((N-1) \frac{\delta}{2}+\delta_{0}\right)\right) .
\end{aligned}
$$

Here, $\delta$ denotes the relative difference in lattice spacing between the film and the substrate; $\delta_{0}$ is the offset at the interface and $N$ is the number of bilayers. $\eta(\leq \sqrt{2}$, depending on the strain) accounts for the buckling within the (111) planes. 


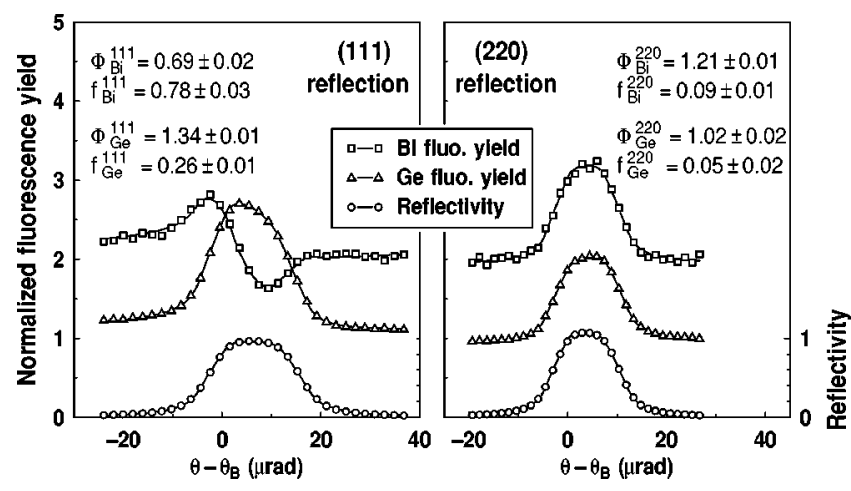

FIG. 1. XSW data obtained after growth of $15 \mathrm{BL}$ Ge on $\mathrm{Bi}: \operatorname{Si}(111)-\sqrt{3}$ $\times \sqrt{3}$ in (111) and (220) Bragg reflection, reflectivity $(\bigcirc), \mathrm{Bi}^{-} \mathrm{L}_{\beta}(\square)$ and $\mathrm{Ge}$ $K \alpha(\triangle)$ fluorescence.

Explanations regarding basic theory and analysis of XSW can be found in the literature. ${ }^{13}$ The output parameters of an XSW analysis are the coherent position $\Phi_{c}$ and coherent fraction $f_{c}$. These are defined as the phase and amplitude of the corresponding Fourier coefficient of the atomic distribution function and for $\mathrm{Ge}$ can hence be calculated from $A_{111}$

$$
\begin{aligned}
& \Phi_{c}^{111}=\frac{1}{2 \pi} \arg \left(A_{111}\right)=\frac{(N-1)}{2} \delta+\delta_{0}, \\
& f_{c}^{111}=\left|A_{111}\right|=\eta\left|\frac{\sin \left(2 \pi N \frac{\delta}{2}\right)}{N \sin \left(2 \pi \frac{\delta}{2}\right)}\right| .
\end{aligned}
$$

If Bi successfully acts as a surfactant then the Bi XSW results should strongly differ from that of Ge. For SME growth, all $\mathrm{Bi}$ is expected to float on the surface and occupy sites at identical height with respect to the surface, depending on the surface roughness. Conclusively, one expects a large $\mathrm{Bi}$ coherent fraction and a small Ge coherent fraction.

Figure 1 displays $\mathrm{Bi}$ and $\mathrm{Ge} \mathrm{XSW}$ data obtained after growth of 15 bilayered (BL) Ge on $\mathrm{Bi}: \operatorname{Si}(111)-\sqrt{3} \times \sqrt{3}$ at $485^{\circ} \mathrm{C}$. The $\mathrm{Bi}$ and $\mathrm{Ge}$ coherent position and fraction are also shown. The results obtained in (111) Bragg reflection are in perfect agreement with the expected values for SME growth. For a completely relaxed Ge film $\left(\delta=0.0423, \delta_{0}\right.$ $\left.\approx 0.02, \eta=\cos (2 \pi(1+\delta) / 8), N=16^{14}\right)$ Eq. (1) yields $\Phi_{c}^{111}$ $=0.34$ (which is physically equivalent to $\Phi_{c}^{111}=1.34$ ) and $f_{c}^{111}=0.28$ in perfect agreement with the experimental results for Ge. In SME, the Bi coherent position is expected to shift according to the number of Ge layers and the difference in (111) lattice plane spacing between Ge and $\mathrm{Si}: \Delta \Phi_{c}^{111}(N)$ $=(N-1) \delta+\delta_{0}+\Delta \Phi_{0}$. From measurements at low Bi coverages ( $\leqslant 1 \mathrm{ML}$ ), we deduce the additional offset due to the Bi-Ge bond length to be $\Delta \Phi_{0}=0.02 \pm 0.01{ }^{11}$ Thus, for $N$ $=16$ we obtain $\Delta \Phi_{c}^{111}=0.69 \pm 0.01$, again perfectly reproducing the experimental results.

The coherent $\mathrm{Bi}$ fraction $f_{\mathrm{Bi}}^{111}$ did not change during growth. After deposition of $15 \mathrm{BL}$ Ge we measured the same value as for the starting $\mathrm{Bi}: \operatorname{Si}(111)-\sqrt{3} \times \sqrt{3}$ surface, i.e., $f_{\mathrm{Bi}}^{111}=0.78 \pm 0.03$. The difference from unity can be attributed to the presence of incoherent $\mathrm{Bi}$ islands on the surface.

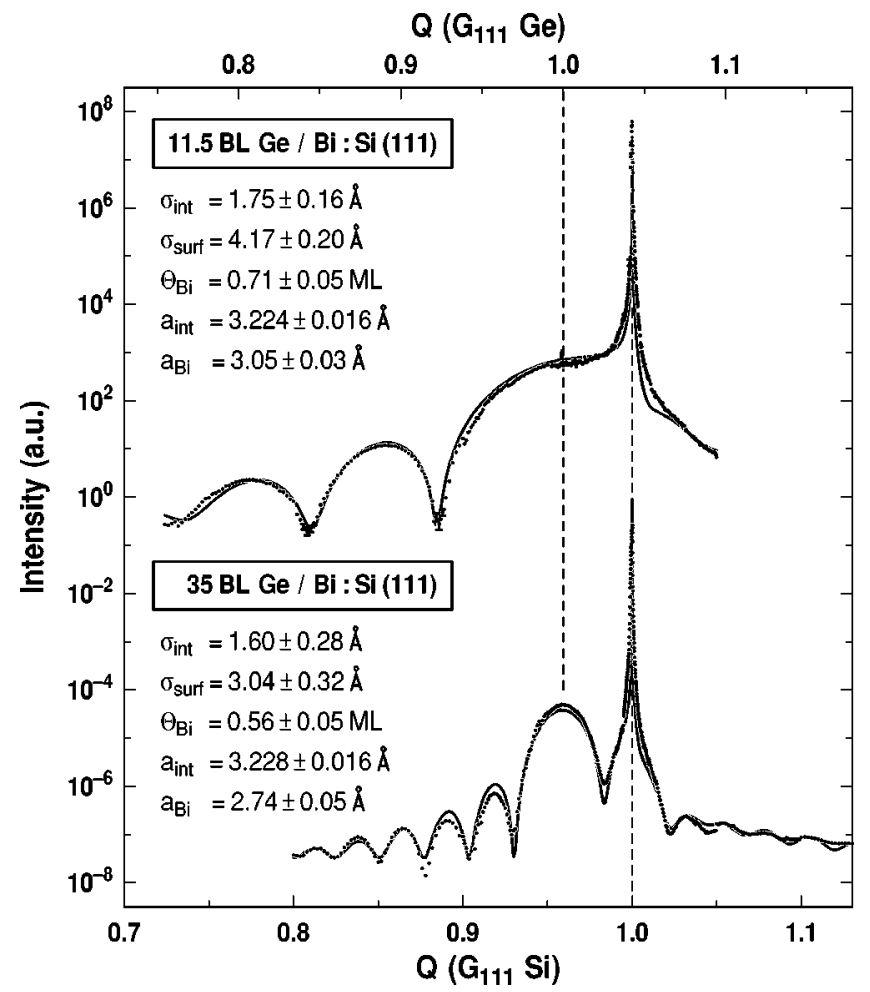

FIG. 2. CTR data ( ) and best fit (solid lines) after deposition of $11.5 \mathrm{BL}$ and $35 \mathrm{BL} \mathrm{Ge}$ on $\mathrm{Bi}: \mathrm{Si}(111)-\sqrt{3} \times \sqrt{3}, Q=1$ corresponds to the (111) Bragg reflection.

The measurements of crystal truncation rods (CTR) is a sensitive tool for the investigation of film thicknesses and the roughness of interfaces and surfaces ${ }^{15,16}$ and has even been successfully employed for the characterization of ultrathin buried Ge structures. ${ }^{17,18}$ Within the kinematical approximation, for a Ge film grown by SME, the CTR intensity can be calculated to be

$$
\begin{aligned}
I(Q)= & \mid F_{\mathrm{Si}}(Q) \frac{\mathcal{R}_{\mathrm{Si}}^{\mathrm{int}}}{1-e^{-i Q a_{\mathrm{Si}}}} \\
& +F_{\mathrm{Ge}}(Q) \frac{\mathcal{R}_{\mathrm{Ge}}^{\mathrm{int}}-\mathcal{R}_{\mathrm{Ge}}^{\mathrm{surf}} \cdot e^{i Q N a_{\mathrm{Ge}}}}{1-e^{i Q a_{\mathrm{Ge}}}} \cdot e^{i Q a_{\mathrm{int}}} \\
& +\left.F_{\mathrm{Bi}}(Q) \Theta_{\mathrm{Bi}} \mathcal{R}_{\mathrm{Ge}}^{\mathrm{surf}} \cdot e^{i Q a_{\mathrm{int}}} e^{i Q N a_{\mathrm{Ge}}} e^{i Q a_{\mathrm{Bi}}}\right|^{2} .
\end{aligned}
$$

The three terms in the model function reflect the sample geometry, i.e., scattering contributions from the Si substrate, the Ge film and the Bi surface layer. The bilayer geometry of the $\mathrm{Si}(111)$ and $\mathrm{Ge}(111)$ planes is found in the factor: $F_{\mathrm{Si}, \mathrm{Ge}}(Q)=f_{\mathrm{Si}, \mathrm{Ge}}(Q) 2 \cos \left((1 / 8) Q a_{\mathrm{Si}, \mathrm{Ge}}\right)$, however $F_{\mathrm{Bi}}(Q)$ $=f_{\mathrm{Bi}}(Q)$. Here $f_{\mathrm{Bi}, \mathrm{Ge}, \mathrm{Si}}(Q)$ denotes the atomic scattering factor of the corresponding element. $N_{\mathrm{Ge}}$ is the number of $\mathrm{Ge}$ layers in the film. For noninteger values of $N$, we used the weighted average of $N=n$ and $N=n+1 ; a_{\mathrm{Si}, \mathrm{Ge}}$ are the lattice spacing of the Si substrate and the Ge layer, respectively. The lattice spacings at the $\mathrm{Si}-\mathrm{Ge}$ interface are denoted as $a_{\mathrm{int}}$. For the lattice spacing between $\mathrm{Ge}$ and $\mathrm{Bi}, a_{\mathrm{Bi}}$ is used. The surface coverage of $\mathrm{Bi}$ is given by $\theta_{\mathrm{Bi}}$. In Eq. 2, both surface and interface roughness are described by a Gaussian distribution function: $\mathcal{R}_{\mathrm{x}}^{\mathrm{y}}=e^{-(1 / 2) q^{2} a_{x}^{2} \sigma_{y}^{2}}$, with $q=Q-2 \pi / a$.

Figure 2 shows CTR measured after deposition of 11.5 (1) 


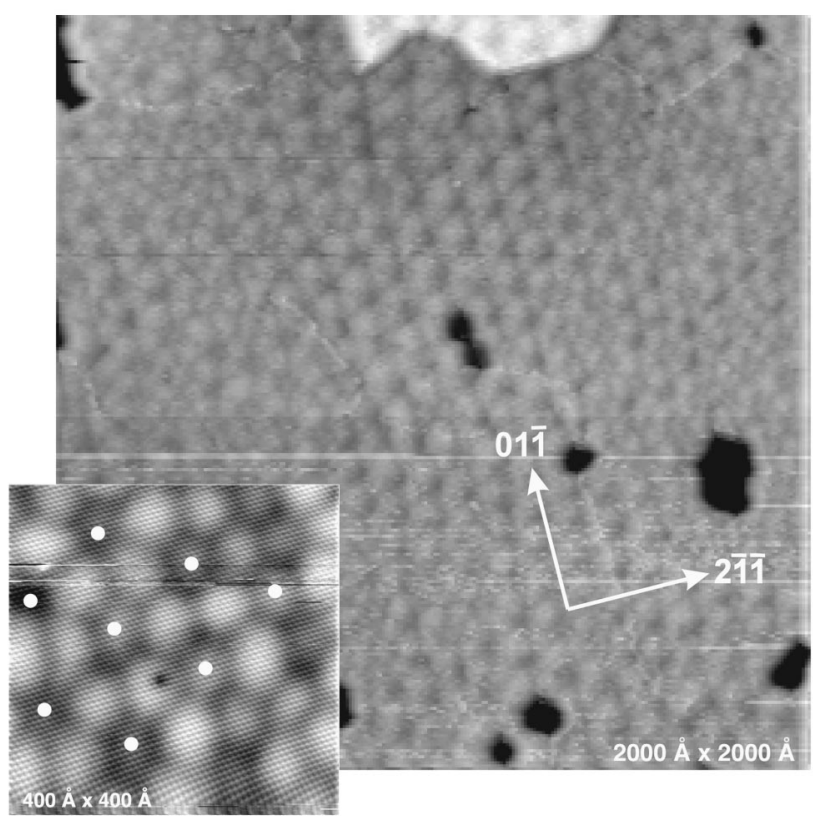

FIG. 3. STM image after deposition of $10 \mathrm{BL}$ Ge on $\mathrm{Bi}: \operatorname{Si}(111)-\sqrt{3} \times \sqrt{3}$ at $485^{\circ} \mathrm{C}$. The inset shows a zoom of $400 \times 400 \AA^{2}$.

and $35 \mathrm{BL}$ Ge on $\mathrm{Bi}: \operatorname{Si}(111)-\sqrt{3} \times \sqrt{3}$ at $485^{\circ} \mathrm{C}$. The results for the major model parameters obtained from least square fits are also displayed. From the fit we determine the Ge lattice spacing to be equal to the bulk lattice spacing of $\mathrm{Ge}$ $a_{\mathrm{Ge}}=a_{\mathrm{Ge}}^{\text {bulk }} \times(1.001 \pm 0.001)$, even after deposition of only $11.5 \mathrm{BL}$ Ge. Hence, at these coverages the Ge film is vertically completely relaxed. We find an interface roughness of 1.6-1.8 $\AA$, which is of the order of the roughness of our starting surface. The surface roughness of the films is found in the order of 3-4 $\AA$, decreasing with film thickness. This effect is due to a rough growth mode for a Ge film thickness around the transition from pseudomorphically strained to relaxed film growth. This process has been investigated in detail by XSW, CTR, and STM and will be the subject of a separate paper. ${ }^{19}$

Figure 3 shows a $2000 \times 2000 \AA^{2}$ STM image obtained after deposition of $10 \mathrm{BL} \mathrm{Ge}$ at $485^{\circ} \mathrm{C}$. Three terrace levels are visible, in good agreement with the small surface roughness determined from the CTR measurements. In addition to single steps at the edges of neighboring terraces a periodic surface height modulation is found in the image with an amplitude of $0.5 \AA$. This feature is even more clearly visible in the inset of Fig. 3 and indicated by superimposing the array of white dots. This frame represents an area of 400 $\times 400 \AA^{2}$ of the same surface. The additional fine structure visible in this image is due to the $\sqrt{3} \times \sqrt{3}$ surface structure, resolved here. The valleys of the periodic surface height modulation (white dots) are aligned in [11 0 ] like orientations and the distance between neighboring rows of minima (indicated by white dots) is approximately $105 \AA$. These observations indicate the presence of a periodic dislocation net- work at the $\mathrm{Si}-\mathrm{Ge}$ interface as also found for $\mathrm{Sb}$ as surfactant. ${ }^{6}$ The formation of this dislocation network enables the relaxation of the pseudomorphically strained $\mathrm{Ge}$ film. The evaluation of the periodicity of the dislocation network shows a laterally completely relaxed Ge film within the experimental uncertainty.

Auger electron spectroscopy (AES) was employed to determine the desorption temperature of $\mathrm{Bi}$ from Ge films on $\mathrm{Si}(111)$. At substrate temperatures exceeding $500{ }^{\circ} \mathrm{C}$ rapid $\mathrm{Bi}$ desorption was observed, resulting in complete removal of $\mathrm{Bi}$ from the surface within two minutes. On bare $\mathrm{Si}(111)$ surfaces the same effect is found at $550^{\circ} \mathrm{C}$. Hence a large $\mathrm{Bi}$ flux is required to compensate for the $\mathrm{Bi}$ desorption during Ge deposition to successfully perform Bi-SME.

In conclusion, $\mathrm{Bi}$ acts as surfactant for $\mathrm{Ge}$ growth on $\mathrm{Si}(111)$. A layer of $\mathrm{Bi}$ segregates to the surface during $\mathrm{Ge}$ deposition and alters the Ge growth from a StranskiKrastanov mode to a layer-by-layer like growth mode for larger amounts of Ge. Measurements of XSW, CTR and STM show that completely relaxed Ge films are found after deposition of more than $10 \mathrm{BL} \mathrm{Ge}$. The relaxation proceeds by the formation of a highly periodic dislocation network at the $\mathrm{Si}-\mathrm{Ge}$ interface. Further $\mathrm{Ge}$ deposition leads to the growth of Ge films with smooth surfaces and interfaces. Most importantly for applications, the surfactant $(\mathrm{Bi})$ can easily be removed from the surface by annealing at temperatures above $500^{\circ} \mathrm{C}$.

${ }^{1}$ M. Copel, M. C. Reuter, E. Kaxiras, and R. M. Tromp, Phys. Rev. Lett. 63, 632 (1989).

${ }^{2}$ M. Horn von Hoegen, F. K. LeGoues, M. Copel, M. C. Reuter, and R. M. Tromp, Phys. Rev. Lett. 67, 1130 (1991).

${ }^{3}$ J. Falta, M. Copel, F. K. LeGoues, and R. M. Tromp, Appl. Phys. Lett. 62 , 2962 (1993).

${ }^{4}$ J. Falta, T. Schmidt, A. Hille, and G. Materlik, Phys. Rev. B 54, R17288 (1996).

${ }^{5}$ S. Maruno, S. Fujita, H. Watanabe, Y. Kusumi, and M. Ichikawa, Appl. Phys. Lett. 68, 2213 (1996).

${ }^{6}$ M. Horn von Hoegen, A. Al Falou, H. Pietsch, B. H. Müller, and M. Henzler, Surf. Sci. 298, 29 (1993).

${ }^{7}$ G. Meyer, B. Voigtländer, and N. M. Amer, Surf. Sci. 274, L541 (1992).

${ }^{8}$ B. Voigtländer and A. Zinner, J. Vac. Sci. Technol. A 12, 1932 (1994).

${ }^{9}$ H. Minoda, S. Sakamoto, and K. Yagi, Surf. Sci. 372, 1 (1997).

${ }^{10}$ J. C. Woicik, G. E. Franklin, C. Liu, R. E. Martinez, I.-S. Hwong, M. J. Bedzyk, J. R. Patel, and J. A. Golovchenko, Phys. Rev. B 50, 12246 (1994).

${ }^{11}$ T. Schmidt, J. Falta, and G. Materlik (unpublished).

${ }^{12}$ E. Vlieg, A. E. M. J. Fischer, J. F. van der Veen, B. N. Dev, and G. Materlik, Surf. Sci. 178, 36 (1986).

${ }^{13}$ J. Zegenhagen, G. Materlik, and W. Uelhoff, J. X-Ray Sci. Technol. 2, 214 (1990).

${ }^{14}$ The lateral atom density of a completely relaxed Ge film compared to that of a pseudomorphically strained Ge film is smaller by a factor of $1 / 1.04^{2}$. Therefore $N \approx 16$ for $\Theta=15 \mathrm{BL}$.

${ }^{15}$ I. K. Robinson, Phys. Rev. B 33, 3830 (1986).

${ }^{16}$ I. K. Robinson, R. T. Tung, and R. Feidenhans'l, Phys. Rev. B 38, 3632 (1988).

${ }^{17}$ J. Falta, D. Bahr, G. Materlik, B. H. Müller, and M. Horn von Hoegen, Appl. Phys. Lett. 68, 1394 (1996).

${ }^{18}$ D. Bahr, J. Falta, G. Materlik, B. H. Müller, and M. Horn von Hoegen, Physica B 221, 96 (1996).

${ }^{19}$ T. Schmidt, J. Falta, and G. Materlik (unpublished). 